\title{
Concordancia del hisopado nasal con el hisopado nasofaríngeo en la detección de virus respiratorios por inmunofluorescencia directa
}

\author{
Paulina Del Pozo, Katia Abarca, Ida Concha y Jaime Cerda
}

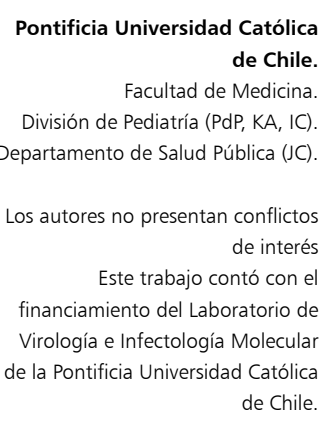

Recibido: 4 de abril de 2013 Aceptado: 4 de febrero de 2014

Correspondencia a: Katia Abarca Villaseca katia@med.puc.cl

\section{Concordance of nasal swabs and nasopharyngeal swabs in the detection of respiratory viruses by direct immunofluorescence}

Background: The most used test for the diagnosis of viral respiratory infection is the detection of viral antigens by direct immunofluorescence (DFA), in samples taken by nasopharyngeal swab (NPS) or aspirate (NPA). It would be desirable to have a less uncomfortable technique to obtain a sample from the patient, but of equal performance. Aim: To evaluate the diagnostic agreement between nasal swab (NS) and nasopharyngeal swab (NPS) in the detection of respiratory viruses by DFA and compare the degree of discomfort of both techniques in pediatric patients. Methodology: Cross-sectional study in children who consulted to a pediatric emergency service with respiratory symptoms. Two samples (NPS and NS) per child were collected. The concordance between the two was determined by Kappa $(\mathrm{K})$ coefficient and the degree of discomfort by a visual pain scale. Results: We obtained 112 samples from 56 children, one by each technique. $82.1 \%$ were concordant, $\mathrm{K}=0.61$ (CI95\%, 0.39-0.83) for the detection of any virus, and $\mathrm{K}=0.69$ (CI 95\%, 0.46- 0.92$)$ and $\mathrm{K}=0.76(\mathrm{CI} 95 \%, 0.51-1)$ for syncytial respiratory virus and influenza A detection, respectively. The degree of discomfort was significantly lower for the NS. Conclusion: There is considerable agreement in the detection of respiratory viruses by DFA between samples obtained by NS and NPS, but not enough to recommend a change in the sampling method in this population.

Key words: Respiratory tract infection, viral disease, diagnostic techniques and procedures, fluorescent antibody technique.

Palabras clave: Infección respiratoria, enfermedad viral, técnicas y procedimientos diagnósticos, Inmunofluorescencia.

\section{Introducción}

$\mathrm{E}$ n Chile, las infecciones respiratorias agudas (IRAs) constituyen un importante problema de salud pública $^{1}$ siendo la principal causa de consulta pediátrica en atención primaria y servicios de urgencia. El $67 \%$ de estas consultas ocurre en niños bajo 6 años de edad ${ }^{2}$, siendo los diagnósticos más frecuentemente consignados IRA alta y síndrome bronquial obstructivo ${ }^{3}$. Las IRAs tienen gran impacto en la morbi-mortalidad mundial infantil ${ }^{4}$ estimándose que representan entre 20 y $40 \%$ de las hospitalizaciones pediátricas en la mayoría de los países en vías de desarrollo ${ }^{5}$, y la primera causa de mortalidad infantil tardía ${ }^{4}$. Las infecciones virales juegan un importante rol como causa de IRA en niños pequeños, asociándose a esa edad con cuadros de mayor gravedad y número de hospitalizaciones ${ }^{6}$. La rápida identificación del agente causal de estas infecciones permite el uso precoz de medidas de control y en algunos casos el inicio de un tratamiento antiviral específico (influenza), reduciendo la posibilidad de infecciones cruzadas, duración de síntomas, estadía hospitalaria y $\operatorname{costos}^{7,8}$.

Actualmente se dispone de múltiples métodos para el diagnóstico de las infecciones respiratorias virales. Si bien el cultivo viral es el estándar de oro ${ }^{9}$, los métodos más utilizados son los de detección de antígenos sumándose en los últimos años las técnicas basadas en biología molecular que han mostrado mayor sensibilidad, pero con la limitación del mayor costo y baja accesibilidad.

La sensibilidad de la detección de antígenos virales a través de inmunofluorescencia directa (IFD), conocido en nuestro medio como "panel viral", depende entre otros factores de una buena recolección de muestra ${ }^{10}$, la que debe contener suficientes células infectadas. Entre las diferentes técnicas de toma de muestra se encuentran el cepillado o hisopado nasofaríngeo (HNF), el aspirado nasofaríngeo (ANF), el lavado nasofaríngeo $(\mathrm{LNF})^{11} \mathrm{y}$, desde hace unos años, el hisopado nasal $(\mathrm{HN})^{12}$, de uso aún no muy difundido y más bien limitado a infecciones virales altas ${ }^{13}$. En la mayoría de los hospitales públicos del país, la técnica 
de toma estándar es el ANF. En nuestra institución, las muestras tomadas por HNF por personal adecuadamente entrenado muestran un adecuado recuento celular y un muy buen rendimiento diagnóstico, con el beneficio adicional de reducir el costo de los insumos utilizados y un menor tiempo de procesamiento de la muestra en el laboratorio, constituyéndose en la toma de muestra estándar. Si bien se ha reportado que el LNF y ANF son más sensibles que el $\mathrm{HNF}$ e $\mathrm{HN}^{3,6,14-18}$, presentan el inconveniente de ser métodos que requieren mayor tiempo de captura, equipamiento especial y entrenamiento del personal que lo realiza. Los tres primeros además causan significativo malestar en el paciente ${ }^{6,10,19}$ y estrés, tanto en el niño como en sus padres, lo que dificulta al personal para su toma adecuada. Por ello, surge la necesidad de explorar alternativas que balanceen la efectividad diagnóstica con el confort del paciente, sin elevar los costos ni los riesgos del procedimiento.

Reportes en la literatura han dado indicios de que el HN sería tan bueno como el ANF en la detección de virus influenza usando inmunofluorescencia (IF) o $\mathrm{ELISA}^{7,8}$; sin embargo, los resultados para el total de los virus no son aún concluyentes dadas las diferentes metodologías empleadas en los distintos estudios. La toma de muestra mediante $\mathrm{HN}$ reduce significativamente la incomodidad para el paciente, por lo que parece ser una alternativa razonable para su uso en la detección de virus respiratorios, siempre que se demuestre que su eficacia diagnóstica es similar a las técnicas de uso estándar. Los objetivos de este trabajo fueron estimar la concordancia del HN con el HNF en la detección de virus respiratorios por IFD y comparar el grado de molestia generado por ambas técnicas, en niños en que se sospecha una infección respiratoria de etiología viral. Se busca con ello evaluar la posibilidad de contar con una metodología de toma de muestra más fácil de realizar por el personal, que provoque menor malestar y por tanto sea más aceptada por el paciente y sus padres, con un rendimiento similar a la utilizada actualmente, pudiendo eventualmente sustituirla.

\section{Pacientes y Métodos}

Se realizó un estudio transversal durante el período comprendido entre los meses de octubre de 2011 y junio de 2012, en que se invitó a participar a todos los niños y niñas entre 0 meses y 15 años de edad que consultaron en el Servicio de Urgencia Pediátrica del Hospital Clínico de la Pontificia Universidad Católica de Chile (PUC) por clínica sugerente de IRA (tos, rinorrea, odinofagia, otalgia, disfonía, estridor o sibilancias, asociadas o no a fiebre) y en quienes el médico tratante solicitó un panel para virus respiratorios por IFD para su diagnóstico. Se excluyó a aquellos pacientes con el antecedente de uso de antivirales anti-influenza durante el mes previo a la consulta o que presentaran alteraciones anatómicas nasales que impidieran la obtención de las muestras. En aquellos pacientes que aceptaron participar, se solicitó la firma de consentimiento informado a uno de los padres o tutor legal del niño y un asentimiento a los niños a partir de los 7 años de edad. El estudio fue aprobado por el Comité de Ética de la Dirección de Investigación de nuestra institución.

Se tomaron dos muestras a cada paciente reclutado, una mediante HNF (método estándar) y otra mediante HN (método en evaluación). Ambas tomas de muestra fueron realizadas por la misma persona, entrenada en la realización de ambos procedimientos. El orden en que se efectuaba la toma de las muestras fue aleatorizado computacionalmente por el método de sobres cerrados por una persona ajena a la investigación. Las muestras fueron rotuladas con un código para mantener el ciego durante su posterior procesamiento.

Para la toma de las muestras, se posicionó al paciente en decúbito supino en posición de olfateo. Para la realización del HNF se introdujo una tórula nasofaríngea de fibra de nylon por una fosa nasal y al tocar la pared faríngea posterior se frotó en la mucosa en forma repetida y se retiró. En el caso del HN se introdujo una tórula nasal de fibra de nylon 2 a $3 \mathrm{~cm}$ desde el exterior en relación al techo nasal de la fosa nasal correspondiente, se giró cerciorándose de frotar la mucosa nasal y se retiró. Cada tórula fue introducida en un tubo con medio de transporte (MTA: $1 \mathrm{ml}$ de tampón fosfato salino $\mathrm{pH}$ $7,2-7,4)$ previamente rotulado con el código asignado y se trasladó en forma refrigerada $\left(2-8^{\circ} \mathrm{C}\right)$ al laboratorio. Se estandarizó que la primera muestra tomada al paciente, independiente del método utilizado para su obtención, siempre se realizara a través de la fosa nasal derecha y la segunda muestra a través de la fosa nasal izquierda.

Adicionalmente y con posterioridad a la toma de cada muestra, se solicitó a los niños entre 4 y 15 años que efectuaran una evaluación del malestar percibido, mediante el uso de la escala de auto-informe de Wong-Baker (que los califica entre 0 y 5 , correspondiendo 5 al dolor o malestar máximo), señalando aquel dibujo que describía mejor cómo se sintió durante el procedimiento realizado para la obtención de la muestra. En el caso de los menores de 4 años, se solicitó a uno de los padres que indicaran usando la misma cartilla su percepción de malestar del niño durante la toma de cada muestra.

Se registraron en una ficha los datos generales del paciente (edad, género, número de identidad-RUT), datos clínicos (signos y síntomas que llevaron a consultar, fecha de inicio de síntomas y fecha de consulta) y la evaluación del malestar del procedimiento.

Las muestras fueron procesadas el mismo día de su toma en el Laboratorio de Virología e Infectología Molecular de la PUC por la técnica de detección de antígenos virales mediante IFD, según el protocolo estándar. Esta 
metodología permite detectar ocho virus respiratorios: virus respiratorio sincicial (VRS), virus influenza A y $\mathrm{B}$, adenovirus, metapneumovirus y virus parainfluenza 1,2 y 3 .

La lectura de las muestras se realizó en forma ciega por personal del laboratorio. Para esto, sólo se procesaron y analizaron en forma inmediata las muestras obtenidas por HNF, postergándose para el final del día el análisis de las muestras obtenidas por $\mathrm{HN}$, de manera que el lector de la IFD no conociera a qué paciente correspondía la muestra obtenida por la técnica a comparar. El informe de laboratorio se entregó en forma habitual a cada paciente con el resultado del examen obtenido por el método de HNF, por ser ésta la técnica estándar en la institución.

Se estimó el tamaño de muestra en 56 pacientes (112

Tabla 1. Virus identificados por IFD en 56 niños usando HN e HNF y concordancia de los resultados entre ambos métodos de toma de muestra

\begin{tabular}{|lccccc} 
Virus aislado & \multicolumn{2}{c}{ Concordancias } & \multicolumn{2}{c}{ Discordancias } & Kappa (IC 95\%) \\
& HNF+/HN+ & HNF-/HN- & HNF+/HN- & HNF-/HN+ & \\
Todos los virus & 32 & 14 & 9 & 1 & $0,61(0,39-0,82)$ \\
VRS & 18 & 14 & 6 & 0 & $0,69(0,46-0,92)$ \\
Adenovirus & 1 & 14 & 0 & 0 & NC \\
Influenza A & 9 & 14 & 3 & 0 & $0,76(0,51-1,00)$ \\
Influenza B & 0 & 14 & 0 & 0 & NC \\
Parainfluenza 1 & 0 & 14 & 0 & 0 & NC \\
Parainfluenza 2 & 0 & 14 & 0 & 0 & NC \\
Parainfluenza 3 & 5 & 14 & 0 & 1 & NC \\
Metapneumovirus & 1 & 14 & 0 & 0 & NC \\
\hline NC: No calculado. & & & & & \\
\hline
\end{tabular}

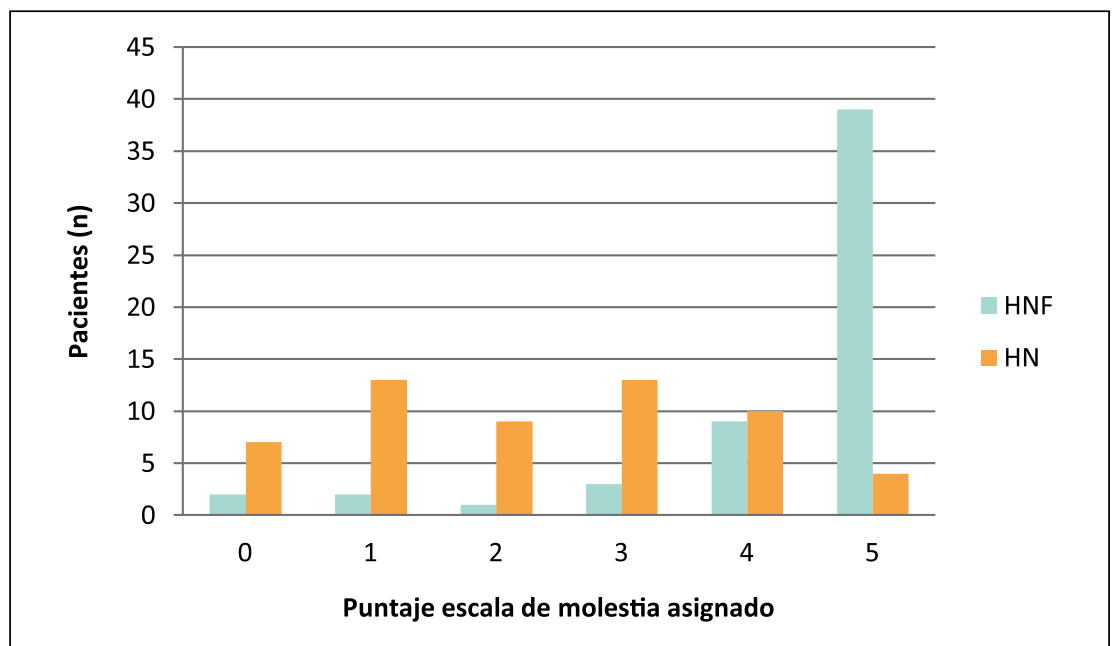

Figura 1. Distribución de puntajes asignados por 56 niños según el grado de molestia percibido con hisopado nasal (HN) e hisopado nasofaríngeo (HNF). $0=$ No duele ni molesta; $5=$ Duele o molesta muchísimo. muestras) para un estudio de concordancia de dos observadores (dos técnicas de toma de muestra en este caso) para detectar un coeficiente de Kappa estadísticamente significativo en el diagnóstico de una variable dicotómica (panel viral positivo versus negativo) ${ }^{21}$, considerándose como supuestos una confianza de $95 \%$, una potencia de $80 \%$ con un Kappa $=0,60$, para una proporción de resultados positivos de $50 \%$ (proporción basada en la experiencia de nuestro centro en junio y julio de 2010 , período en que la proporción de paneles positivos fue 54,9\%). Se estimó la concordancia general entre ambos métodos para todos los virus respiratorios presentes en el panel viral y para aquellos virus encontrados en al menos 10 pacientes. Se utilizó la valoración de los distintos grados de concordancia según el resultado del coeficiente Kappa de Landis y $\mathrm{Koch}^{22}$, que indica que para un coeficiente Kappa entre 0,21 y 0,40 la concordancia se considera aceptable; entre 0,41 y 0,60 moderada; entre 0,61 y 0,80 , considerable; y entre 0,81 y 1,00 óptima.

\section{Resultados}

Se reclutó un total de 56 pacientes de los cuales se obtuvo 112 muestras para ser analizadas por IFD (56 obtenidas por HNF y 56 por HN). El promedio de edad de los pacientes fue de 32,5 meses $(0-172) ; 64,2 \%$ de los sujetos eran de género masculino. El promedio de días de síntomas al momento de la consulta fue de 4,8 días (021). Los principales síntomas por los cuales consultaron los pacientes correspondieron a tos $(96,4 \%)$, rinorrea $(92,8 \%)$, y fiebre $(80,3 \%)$.

De los 56 pacientes, en 41 (73\%) se identificó algún virus respiratorio en muestras tomadas por HNF y en 33 (59\%) en las tomadas por $\mathrm{HN}$.

Con estos resultados de detección viral obtenidos por los dos métodos, se determinó que las muestras obtenidas por ambas técnicas fueron concordantes en $82,1 \%$ de los casos, calculándose un coeficiente de Kappa de 0,61 (IC 95\% 0,39-0,82) para la detección de cualquier virus respiratorio, de 0,69 (IC 95\% 0,46- 0,92) para la detección de VRS y de 0,76 (IC 95\% 0,51-1) para el virus influenza A. No se analizó la concordancia para los otros virus debido al bajo número de pacientes con presencia de tales virus. La distribución de concordancias y discordancias obtenidas para ambos métodos se resumen en la Tabla 1.

En relación a la evaluación del malestar asociado a la toma de muestra por el paciente o alguno de los padres a través de la escala visual de dolor, la mediana de la puntuación obtenida fue de 5 para las muestras obtenidas por HNF y de 2 para las muestras obtenidas por $\mathrm{HN}$, siendo esta diferencia estadísticamente significativa $(\mathrm{p}<0,001)$. El gráfico muestra la distribución de puntajes de molestia percibidos para cada técnica de toma de muestra (Figura 1). 


\section{Discusión}

El presente estudio demostró una concordancia considerable (de acuerdo a la clasificación de Landis y Koch) entre dos técnicas de obtención de muestra (HNF e HN) para la detección diagnóstica de cualquier virus respiratorio mediante el uso de IFD en la muestra de pacientes estudiados. Al analizar según virus específico, la concordancia mejora en el caso de detección de VRS y de influenza A, sin llegar a ser óptima.

Una limitación de este estudio radica en no haberse obtenido un número de muestras positivas para cada uno de los virus que lograra ser representativo y adecuado para el cálculo del índice Kappa para cada uno de ellos. Esto debido a que el tamaño de muestra se calculó en general y no para cada virus en particular, y a la epidemiología variable de las infecciones virales respiratorias durante el transcurso del año que limitó el número de muestras positivas para algunos virus en el período acotado de tiempo en que se realizó el estudio.

Por estos motivos esta investigación deba ser considerada como un estudio preliminar, que puede motivar la realización de estudios futuros. La tendencia de los resultados sugiere que un estudio de mayor extensión temporal y con mayor número de muestras recolectadas, calculadas en base a la prevalencia de cada virus analizado, podría demostrar una concordancia adecuada que eventualmente permitiera recomendar el reemplazo de las técnicas de toma de muestra utilizadas actualmente.

Los resultados indican una clara ventaja del $\mathrm{HN}$ en relación al HNF en cuanto al malestar provocado en los niños. Además, el HN es una técnica de toma de muestra accesible, fácil de aprender y reproducir por el personal de salud y que no involucra mayores costos en insumos o mayor trabajo de laboratorio que el que actualmente se requiere para la realización del panel viral, puesto que las tórulas utilizadas son de características similares a las usadas para el HNF y la metodología de análisis es la misma.

Si bien la concordancia obtenida fue considerable, estimamos que no es suficientemente alta como para recomendar el reemplazo del método de obtención de muestras respiratorias usado en nuestra institución (HNF). Una de las razones tiene relación con el rango del intervalo de confianza que presenta el coeficiente de concordancia kappa $(0,39-0,83)$, el que es bastante amplio, de modo que en el peor escenario la concordancia diagnóstica sería solamente aceptable (de acuerdo a la clasificación de Landis y Koch). Una segunda razón radica en el análisis de las discordancias observadas: existen nueve casos de discordancia $\mathrm{HN}-/ \mathrm{HNF}+$ y sólo un caso de discordancia $\mathrm{HN}+/ \mathrm{HNF}$-. Estos resultados demuestran que la IFD realizada en muestra obtenida por HNF tiene mayor probabilidad de resultar positiva en comparación a la IFD realizada en muestra obtenida por $\mathrm{HN}$, brindando una mayor probabilidad de acceder a un diagnóstico virológico.

Si bien los resultados obtenidos en este estudio no permiten hacer una recomendación de reemplazar el uso actual del HNF por el HN para la detección de virus respiratorios mediante IFD, sí permiten plantear a esta técnica de toma de muestra, mejor tolerada por los niños, como una alternativa a analizar para el diagnóstico virológico con una metodología de mayor rendimiento como es la amplificación de ácidos nucleicos mediante reacción de polimerasa en cadena (RPC). Esta metodología actualmente ha ido reemplazando al cultivo viral y a la IFD por su elevada sensibilidad y, al ir reduciendo sus costos, cada vez se encuentra más disponible y accesible para los pacientes.

Agradecimientos. A los pacientes y sus familias que aceptaron participar de este estudio. Al personal médico y de enfermería del Servicio de Urgencia Pediátrica del Hospital Clínico de la Pontificia Universidad Católica de Chile. Al personal técnico del Laboratorio de Virología e Infectología Molecular de la Pontificia Universidad Católica de Chile.

\section{Resumen}

Introducción: El examen más utilizado para el diagnóstico de infecciones respiratorias virales es la detección de antígenos virales por inmunofluorescencia directa (IFD) en muestras tomadas por hisopado nasofaríngeo (HNF) o aspirado (ANF). Sería deseable contar con una técnica de toma de muestra menos molesta para el paciente pero de igual rendimiento que las mencionadas. Objetivo: Evaluar la concordancia diagnóstica entre hisopado nasal (HN) e HNF en la detección de virus respiratorios por IFD y comparar el grado de molestia de ambas técnicas en pacientes pediátricos. Métodos: Estudio transversal en niños que consultaron en una urgencia pediátrica por síntomas respiratorios agudos. Se recolectaron dos muestras (por HNF y HN) por cada niño para detección viral mediante IFD. Se determinó la concordancia entre ambas mediante coeficiente Kappa (K) y se analizó el grado de molestia que causa la toma de muestra con escala visual. Resultados: Se obtuvo 112 muestras en 56 niños, una por cada técnica. La concordancia entre ellas fue de $82,1 \%$, con un $\mathrm{K}=0,61$ (IC95\% 0,39-0,83) para la detección de cualquier virus, $\mathrm{K}=0,69$ (IC 95\% 0,46- 0,92) para virus respiratorio sincicial y $\mathrm{K}=0,76$ (IC 95\% $0,51-1$ ) para influenza A. El grado de molestia fue significativamente menor para el HN. Conclusiones: Existe una concordancia considerable en la detección de virus respiratorios mediante IFD en muestras tomadas por HNF como HN, pero insuficiente como para recomendar un cambio de metodología de la toma de muestra en esta población. 


\section{Referencias bibliográficas}

1.- Girardi G, Astudillo P, Zúñiga F. El programa IRA en Chile: hitos e historia. Rev Chil Pediatr 2001; 72: 292-300.

2.- López I M, Sepúlveda H B, Guerra J F, Nazar R, Valle P. Perfil de morbilidad anual de menores de 6 años consultantes. Rev Chil Pediatr 2000; 71: 321-7.

3.- Méndez B, Herrera P, Guerra H, Dattas J P, Muñoz B, Velasco J. Estructura de la consulta pediátrica en el Servicio de Urgencia. Hospital Infantil Roberto del Río. Rev Chil Pediatr 2005; 76: 259-65.

4.- Avendaño L F, Céspedes A, Stecher X, Palomino M A. Influencia de virus respiratorios, frío y contaminación aérea en la infección respiratoria aguda baja del lactante. Rev Med Chile 1999; 127: 1073-8.

5.- Barría R M, Calvo M. Factores asociados a infecciones respiratorias dentro de los tres primeros meses de vida. Rev Chil Pediatr 2008; 79: 281-9.

6.- Bosis S, Esposito S, Niesters H G. Role of respiratory pathogens in infants hospitalized for a first episode of wheezing and their impact on recurrences. Clin Microbiol Infect 2008; 14 677-84.

7.- Heikkinen T, Salmi A A, Ruuskanen O. Comparative study of nasopharyngeal aspirate and nasal swab specimens for detection of influenza. Br Med J 2001; 322: 138.

8.- Ipp M, Carson S, Petric M, Parkin P C. Rapid painless diagnosis of viral respiratory infection. Arch Dis Child 2002; 86: 372-3.
9.- Schutzbank T E, McGuire R, Scholl D R. Immunofluorescence. Specter S, Hodinka RL, Young SA, Wiedbrauk DL, Clinical Virology Manual, fourth ed. Washington: ASM Press; 2009, p. 77-88.

10.- Faden H. Comparison of midturbinate flockedswab specimens with nasopharyngeal aspirates for detection of respiratory viruses in children by the direct fluorescent antibody technique. J Clin Microbiol 2010; 48: 3742-3.

11.- Barnes S D, Leclair J M, Forman M S, Townsend T R, Laughlin G M, Charache P. Comparison of nasal brush and nasopharyngeal aspirate techniques in obtaining specimens for detection of respiratory syncytial viral antigen by immunofluorescence. Pediatr Infect Dis J 1989; 8: 598-601.

12.- Sung R Y, Chan P K, Choi K C, Yeung A C, Li AM, Tang JW, et al. Comparative study of nasopharyngeal aspirate and nasal swab specimens for diagnosis of acute viral respiratory infection. J Clin Microbiol 2008; 46: 3073-6.

13.- Ginocchio C C, McAdam A. Current best practices for respiratory virus testing. J Clin Microbiol 2011; 49 (9 Suppl): S44-8.

14.- Ahluwalia G, Embree J, McNicol P, Law B, Hammond G W. Comparison of nasopharyngeal aspirate and nasopharyngeal swab specimens for respiratory syncytial virus diagnosis by cell culture, indirect immunofluorescence assay, and enzyme-linked immunosorbent assay. J Clin Microbiol 1987; 25: 763-7.

15.- Frayha H, Castriciano S, Mahony J, Chernesky M. Nasopharyngeal swabs and nasopharyngeal aspirates equally effective for the diagnosis of viral respiratory disease in hospitalized children. J Clin Microbiol 1989; 27: 1387-9.

16.- Heikkinen T, Marttila J, Salmi AA, Ruuskanen O. Nasal swab versus nasopharyngeal aspirate for isolation of respiratory viruses. J Clin Microbiol 2002; 40: 4337-9.

17.- Macfarlane P, Denham J, Assous J, Hughes C. RSV testing in bronchiolitis: which nasal sampling method is best? Arch Dis Child 2005; 90: 634-5.

18.- Stensballe L G, Trautner S, Kofoed P E, Nante E, Hedegaard K, Jensen I P, et al. Comparison of nasopharyngeal aspirate and nasal swab specimens for detection of respiratory syncytial virus in different settings in a developing country. Trop Med Int Health 2002; 7: 317-21.

19.- Abu-Diab A, Azzed M, Ghneim R, Ghneim R, Zoughbi M, Turkuman S, et al. Comparison between pernasal flocked swabs and nasopharyngeal aspirates for detection of common respiratory viruses in samples from children. J Clin Microbiol 2008; 46: 2414-7.

20.- Tomlinson D, Von Baeyer CL, Stinson JN, Sung L. A systematic review of faces scales for the self-report of pain intensity. Pediatrics 2010; 126: 1168-98.

21.- Sim J, Wright C C. The kappa statistic in reliability studies: use, interpretation, and sample size requirements. Phys Ther 2005; 85: 257-68.

22.- Cerda J, Villarroel L. Evaluación de la concordancia inter-observador en investigación pediátrica: Coeficiente de Kappa. Rev Chil Pediatr 2008; 79: 54-8. 